\title{
Is Cardiolipin the Target of Local Anesthetic Cardiotoxicity?
}

\author{
XiaoFeng Shen ${ }^{4}$, FuZhou Wang, TSA 2, ShiQin Xu ${ }^{3}$, YanNing Qian, TSA 4, YuSheng Liu ${ }^{5}$, HongMei Yuan ${ }^{6}$, QingSong Zhao ${ }^{6}$, \\ ShanWu Feng ${ }^{5}$, XiRong Guo, TSA ${ }^{7}$, JianGuo Xu, TSA ${ }^{8}$, Jie Yang, TSA ${ }^{9}$
}

\begin{abstract}
Summuary: Shen X, Wang F, Xu S, Qiang Y, Liu Y, Yuan H, Zhao Q, Feng S, Guo X, Xu J, Yang J - Is Cardiolipin the Target of Local Anesthetic Cardiotoxicity?

Background and objectives: Local anesthetics are used broadly to prevent or reverse acute pain and treat symptoms of chronic pain. Local anesthetic-induced cardiotoxic reaction has been considered the accidental event without currently effective therapeutic drugs except for recently reported intralipid infusion whose possible mechanism of action is not well known.
\end{abstract}

Contents: Cardiolipin, an anionic phospholipid, plays a key role in determining mitochondrial respiratory reaction, fatty acid metabolism and cellular apoptosis. Mitochondrial energy metabolism dysfunction is suggested as associated with local anesthetic cardiotoxicity, from an in vitro study report that the local anesthetic cardiotoxicity may be due to the strong electrostatic interaction of local anesthetics and cardiolipin in the mitochondria membrane, although there is a lack for experimental evidence. Herein we hypothesized that local anesthetic-cardiolipin interactions were the major determinant of local anesthetic-associated cardiotoxic reaction, established by means of theoretic and structural biological methods. This interacting model would give an insight on the underlying mechanism of local anesthetic cardiotoxicity and provide clues for further in depth research on designing preventive drugs for such inadvertent accidence in routine clinical practice.

Conclusions: The interaction between local anesthetic and mitochondrial cardiolipin may be the underlying mechanism for cardiotoxicity affecting its energy metabolism and electrostatic status.

Keywords: ANESTHETICS, Local; CARDIOLIPINS; COMPLICATIONS, Arrhythmias; cardiac, heart arrest, induced.

Financial Suport: This work has been supported in part by the grant of Science and Technology Development of Nanjing Medical University (07NMUM065, 08NMUZ033) and the National Natural Science Fund of China (NSFC, 30901397) to FW.

[Rev Bras Anestesiol 2010;60(4): 445-454] CElsevier Editora Ltda.

\section{INTRODUCTION}

Local anesthetics are one of the major components of modern Anesthesiology. Local anesthetic-associated cardiotoxicity is,

Received from the Department of Anesthesiology, of the Affiliated Nanjing Maternity and Child Health Care Hospital, Nanjing Medical University and State Key Laboratory of Pharmaceutical Biotechnology, Nanjing University, Nanjing, China.

1. Professor; Director of Anesthesiology Department of the Affiliated Nanjing Maternity and Child Health Care Hospital, Nanjing Medical University

2. Doctor; Senior Lecturer, Research Consultant; Affiliated Nanjing Maternity and Child

Health Care Hospital, Nanjing Medical University; State Key Laboratory of Pharmaceutical

Biotechnology, School of Life Science, Nanjing University

3. Associate Professor; Staff Anesthesiologist of the Affiliated Nanjing Maternity and Child Health Care Hospital, Nanjing Medical University

4. Professor; Co-Chairman of Jiangsu Association of Anesthesiology; Department of Anes-

thesiology, of the First Affiliated Hospital, Nanjing Medical University

5. Lecturer - Staff Anesthesiologist of the Affiliated Nanjing Maternity and Child Health Care Hospital, Nanjing Medical University

6. Student of the Affiliated Nanjing Maternity and Child Health Care Hospital, Nanjing Medical University

7. Professor; Chairman of the Institute of Pediatrics; Affiliated Nanjing Maternity and Child Health Care Hospital, Nanjing Medical University

8. Professor; Co-Chairman of Chinese Association of Anesthesiology; Department of Anes-

thesiology, Jingling Hospital, Medical School of Nanjing University

9. Professor-Director of Bioinformatics Department; State Key Laboratory of Pharmaceuti-

cal Biotechnology, School of Life Science, Nanjing University

Submitted on October 1, 2009

Accepted on March 18, 2010

Correspondence to:

Dr. FuZhou Wang

Department of Anesthesiology

Affiliated Nanjing Maternity and Child Health Care Hospital

Nanjing Medical University, 123

Tianfei Xiang, Mochou Road

Nanjing 210004 - China

E-mail: zfwang50@njmu.edu.cn however, a thorny concern which causes atrioventricular block and asystole threatening the patients' life. While several reports gave a promising view regarding the successful postaccidental resuscitation of local anesthetic cardiotoxicity with intralipid ${ }^{1-6}$, critical debates still exist for this controversial issue ${ }^{7-12}$. This may be related to unclear mechanisms of action for local anesthetics on cardiac myocytes.

Mitochondrial respiratory chain is the center of energy metabolism in cellular activities by synthesizing adenosine triphosphate (ATP) through oxidative phosphorylation, that can be changed or blocked or even destroyed in numerous pathological conditions. Cumulating evidence indicates that local anesthetics have diverse effects on oxidative phosphorylation in mitochondria largely depending on their chemical structures. Although such strong association between local anesthetic and mitochondria was documented, the precise role for local anesthetics in cardiac mitochondrial oxidative phosphorylation is yet unknown. Given that local anesthetic cardiotoxicity is a key part of their systemic toxicity, it is necessary to explore the potential mechanisms of local anesthetics on mitochondria metabolism in cardiac myocytes.

Cardiolipin, a unique tetra-acyl phospholipid firstly isolated from beef heart in the early 1940s is found almost exclusively in the inner mitochondrial membrane where it is essential for the optimal function of numerous enzymes that are involved in mitochondrial energy metabolism ${ }^{25}$. Alterations in the content and/or structure of cardiolipin have been reported in several 
tissues in a variety of pathological settings, such as Barth syndrome, ischemia and reperfusion, aging, thyroid status, heart failure, neurodegenerative disease, dietary 18:2 deficiency, chronic ethanol consumption, and diabetes (detailed contents, see review 26). Önyüksel and colleagues showed that bupivacaine, but not lidocaine, interacts avidly and selectively with biomimetic small unilamellar liposomes containing cardiolipin and disrupts their integrity, which suggested that this interaction underlies in part the bupivacaine-induced cardiotoxicity ${ }^{27}$. Cardiolipin functions as a key mediator of mitochondrial metabolism ${ }^{25}$. Considering some previous reports on successful resuscitation after inadvertent intravenous administration of local anesthetics by means of lipid emulsion, we proposed, to this end, that cardiolipin could take an essential part in local anesthetic-induced cardiotoxicity.

We hereby hypothesized that the interaction between cardiolipin and local anesthetics is the underlying mechanism of local anesthetic cardiotoxicity considering a developed computational model.

\section{Local anesthetic cardiotoxicity}

The main clinical manifestation of systemic toxic reactions to local anesthetics follows the increasing in blood levels of local anesthetics ${ }^{28-30}$. Initially, symptoms from central nervous system (CNS) excitation are described including a metallic taste in the mouth, a ringing in the ears, or a circumoral tingling. Progressively, motor twitching in the periphery is followed by grand mal seizures, coma and respiratory arrest. Terminally, cardiac arrhythmia, hypotension, cardiovascular collapse and asystole occur.

Cardiotoxicity is the focus of local anesthetic-associated systemic toxic reactions mainly because the difficulty in achieving success in resuscitation after such an inadvertent accidence. Given the ethical concern, it is nearly impossible to perform clinical studies to clarify the precise association between cardiotoxicity and local anesthetics. Therefore, case reports and case series are the major clinical literatures in assessing the prognosis and frequency of such events. Multiple case reports of cardiac arrest and electrical standstill appeared after local anesthetic toxicity, and a large number were associated with difficult resuscitation, especially in the obstetrical population ${ }^{31,32}$. To these patients, even a cardiopulmonary bypass was used as a therapeutic choice ${ }^{33}$. Recently, several case reports documented successful resuscitation after lipid emulsion infusion ${ }^{1,4,5}$, whereas its actual role in reducing the mortality has been intensely discussed ${ }^{7-12}$. However, it is still not an optimistic issue for patients and medical caregivers when using local anesthetics, especially the lipophilic amides, in case of the accidental intravenous injection or large dose of them reaching the toxic levels.

In reality, it is difficult to assess the frequency of local anesthetic-induced cardiotoxcity due to the lack of large-scale and strong evidence-based studies. A theoretic method created by Hanley and Lippman-Hand could estimate the risk of an accidental event that has not occurred in a prospective series ${ }^{34}$, in which the upper limit of the predicted probability with a onesided $95 \%$ confidence interval $(95 \% \mathrm{Cl})$ is three to $\mathrm{n}$, where $\mathrm{n}$ is the number of observations without an adverse event. For example, if the $n=60$, then the maximum risk of cardiotoxicity after systemic toxic reaction would be five per cent.

In consideration of the poor prognosis of local anesthetic cardiotoxicity combining the difficult prediction of the emergence frequency, further studies on the interaction mechanisms between local anesthetics and cardiotoxic reaction are need. Furthermore, theoretic and computational methods may be the better ways when clinical studies are restricted.

\section{Cardiolipin and mitochondrion}

Cardiolipin plays a crucial role in the mitochondrial membrane-stabilization, formation of the protein supercomplexes and functioning of respiratory chain. Cardiolipin is required for the electron transfer process in complex I and III of mitochondrial respiratory chain and structural organization of the complexes III and IV into a supercomplex. It stabilizes respiratory chain supercomplexes as well as the individual complexes, and is required to prevent formation of the resting state of cytochrome c oxidase in the membrane. Cardiolipin functions as an effector of activity of a mitochondrial cytochrome $\mathrm{p}-450^{35}$, and may contribute to the efficiency of oxidative phosphorylation both by decreasing the distance through which cytochrome $\mathrm{c}$ must travel between complexes III and IV as well as placing ADP/ ATP carrier (AAC) in an environment that promotes its optimal activity ${ }^{36}$. In addition, the novel mitochondria-vacuole signaling pathway is mediated by the synthesis of cardiolipin ${ }^{37}$. The mitochondrial creatine kinase, a water-soluble octamer-dimer enzyme bound to the cytoplasmic side of the inner mitochondrial membrane, exerts functions via binding to cardiolipin as its receptor ${ }^{38}$. Taken these information together, cardiolipin has been regarded as the heart of mitochondrial metabolism for the importance of cardiolipin content and composition in various common diseases, such as diabetes and heart failure 25,39,40. This role determined that detailed analyses on cardiolipin are needed for patients suffering from mitochondrial disease with unknown origin, since cardiolipin abnormalities might be their underlying cause.

\section{Local anesthetics and mitochondrion}

In in vitro isolated mitochondria, local anesthetics have diverse effects on oxidative phosphorylation depending on their chemical structures, including inhibiting electron transport ${ }^{13,14}$ and $\mathrm{F}(1)$ ATPase ${ }^{15-17}$, affecting transport of ions such as $\mathrm{Ca}^{2+18-21}$, and causing uncoupling 22,23 . Terada and colleagues found that the action of bupivacaine on mitochondria was mainly through accelerating the state-four respiration and activating the ATPase without the dissipation of the proton electrochemical potential where lacking hydrophobic anions, i.e., a decoupling action but not uncoupling was brought about ${ }^{24}$. The property of lipid solubility ${ }^{41}$ but not the stereospecific effects ${ }^{42}$ of lo- 
cal anesthetics determines their effects on mitochondrial bioenergetics. Ropivacaine is less potent than bupivacaine on mitochondrial bioenergetics due to its lower lipid solubility in isolated rat heart mitochondria and in saponin-permeabilized ventricular fibers, and this cardiac energy metabolism impairment of bupivacaine can be enhanced with chronic hypoxia ${ }^{43}$.

In cell culture, local anesthetics can reach mitochondria and reversibly decrease, or even collapse, their transmembrane potential ${ }^{44}$. The disruption of $\mathrm{Ca}^{2+}$ homeostasis in vivo has been suggested contributing to the bupivacaine toxicity 45,46 . Besides, mitochondrial dysfunction results in ATP depletion 47 and in turn is expected to have a major impact on intracellular $\mathrm{Ca}^{2+}$ homeostasis ${ }^{48}$. In addition, active oxidative metabolism is a key determinant in bupivacaine toxicity, and bupivacaine myotoxicity is a relevant model of mitochondrial dysfunction involving the permeability transition pore (PTP), a cyclosporin A-sensitive inner membrane channel that plays a key role in many forms of cell death, and $\mathrm{Ca}^{2+}$ dysregulation, and that it represents a promising system to test new PTP inhibitors that may prove relevant in spontaneous myopathies where mitochondria have long been suspected to play a role ${ }^{49}$. Longacting local anesthetics induce marked negative inotropic and lusitropic effects on cardiomyocytes and such effect was mainly because of the impairment of calcium handling ${ }^{50}$. To the regulation of $\mathrm{Ca}^{2+}$ homeostasis, different local anesthetics possess different functions; bupivacaine, levobupivacaine, and ropivacaine can induce a deleterious effect in mitochondrial energy, though levobupivacaine disturbs $\mathrm{Ca}^{2+}$ homeostasis in the greatest degree ${ }^{51}$.

Additionally, mitochondrial apoptotic pathway was considered as an essential part in leading to local anesthetic-induced cardiotoxicity. Mitochondrial swelling and oxidation of membrane protein thiol groups were associated with the activation of PTP, which was inhibited by the local anesthetic ${ }^{52}$. DNA fragmentation and DNA "ladder" formation, a typical feature for apoptosis, were induced by dibucaine with half-maximal concentration of $100 \mu \mathrm{M}$, and these effects were completely prevented by the unspecific caspase inhibitor z-Val-Ala-Asp(OMe)-fluoromethylketone, thereby implicating caspase activation in the process ${ }^{53}$. Lidocaine-induced apoptosis was also associated with the mitochondrial caspase pathways ${ }^{54}$. Whether there are any interacting relationship among local anesthetics, caspase activation, apoptosis and cardiac toxic reaction still need further studies.

Recent reports ${ }^{1-6,55}$ upon successful resuscitation with lipid emulsion after local anesthetic-induced cardiac collapse proposed that lipid metabolism might take a crucial part in explaining the mechanisms of the successfully resuscitated heart. Fatty acid metabolism was shown to predispose the isolated heart to bupivacaine toxicity, which confirmed that the local anesthetic exerts specific effects on lipid processes in cardiomyocytes ${ }^{56}$. Given the mitochondrion is the major compartment of lipid metabolism in living cells, and the socalled activation of fatty acids that is the obligatory step in fatty acid metabolism occurs partly in the outer mitochondrial membrane (for long-chain fatty acids) or in the mitochondrial matrix (for medium-chain fatty acids), the abnormal metabo- lism of fatty acids in heart mitochondria after local anesthetics overdosage should be explored in depth.

\section{Local anesthetics and cardiolipin}

Cardiolipin as described above has been recognized as a key mediator of the mitochondrial oxidative phosphorylation ${ }^{35-38}$, phospholipid metabolism ${ }^{57-59}$, and cellular apoptosis 60,61 . The in vitro study found that bupivacaine interacts selectively with biomimetic small unilamellar liposomes containing cardiolipin and disrupts the liposome integrity, suggesting that the bupivacaine-cardiolipin interaction might be the underlying mechanism of bupivacaine-induced cardiotoxicity ${ }^{27}$. In addition to this implication, further investigations proved indirectly that (i) anionic, pegylated liposomes (polyethylene glycol (PEG) attached, at one end of the polymer chain, to the surface of liposomes) exhibit high binding for bupivacaine ${ }^{1}$, (ii) liposomes containing cardiolipin possess property of membrane fluidity ${ }^{62}$, (iii) local anesthetics accelerate the water permeability by destabilizing the membrane structure and this effect was governed by the hydrophobicity of the anesthetics ${ }^{63}$, and (iv) a minimal $\mathrm{Ca}^{2+}$ concentration is required for the fusion of large $(0.1 \mu \mathrm{m})$ unilamellar cardiolipin/phosphatidylcholine (1:1) vesicles monitored by the mixing of the aqueous contents ${ }^{64}$. Currently, local anesthetics are used as model drug compounds when investigating the mechanism of drug release from oil suspensions in vitro ${ }^{65}$, whereas the cardiotoxicity of local anesthetics evoked by interacting with mitochondrial cardiolipin or not is yet to be known. Besides, whether such theoretic interaction between the local anesthetic and cardiolipin played an expected role in local anesthetic-induced cardiac toxic reaction, it has to be explored in depth with experiments and theoretic models.

\section{Hypothesis - the interacting model of cardiolipin and local anesthetics}

Interacting model established by using computational theories became one of the pivotal means of theoretic biology. Cardiolipin-related interaction models have been developed such as the investigation of cardiolipin and its effect on the structure of lipid bilayers with molecular dynamics computer simulations ${ }^{66}$, the formation of the cytochrome c-cardiolipin complex by ionic strength ${ }^{67}$, mathematical model of pattern formation of molecular species of mitochondrial cardiolipin ${ }^{58}$, and cardiolipin's electrostatic locking role in the Bax-alpha1 targeting sequence interact with mitochondrial membranes ${ }^{68}$. While numerous models of cardiolipin has been developed, the interacting model of the local anesthetic with cardiolipin has so far not been established especially when discussing the negative effect of local anesthetics on cardiac activity.

Muhonen et al. studied the interactions between local anesthetics and lipid dispersions using liposome electrokinetic capillary chromatography (LEKC), and found that all tested anesthetics (bupivacaine, lidocaine and prilocaine) that once 
attached to negatively charged cardiolipin molecules in the liposomes by electrostatic forces could not be released from the intralipid particles anymore ${ }^{69}$. Even so, the precise interacting relation between local anesthetics and cardiolipin is still not known. Methods used in computational biology, such as molecular docking and packing, quantitative structure-activity relationships (QSAR), Monte Carlo simulated annealing approach, structural bioinformatics, pharmacophore modeling, and signal peptide prediction, among others, can effectively provide bioinformation and insights into corresponding molecule-molecule interactions. Herein we hypothesized that the interacting model between the local anesthetic and mitochondrial cardiolipin suggests a key role in determining the cardiac toxic reaction of local anesthetics, and such model can be developed with aforementioned methods to predict the cardiotoxicity of the local anesthetic.

\section{CONCLUDING REMARKS AND CLINICAL IMPLICATIONS}

Not merely the inadvertent injection of local anesthetics into blood vessels, but the sustained and prolonged duration of local anesthesia ${ }^{70}$ can result in toxic reaction. How to reduce or prevent the local anesthetic-induced cardiotoxicity is an essential issue for clinical professionals when they used local anesthetics routinely. Nonetheless, under the condition of effectively preventive strategic guidelines, the reliability and safety of drugs to reverse the cardiotoxic reaction are very important in the event of an accident. It is necessary a detailed and clear understanding of the underlying mechanisms of local anesthetic cardiotoxicity.

In view of recently reported successful cases resuscitated with intralipid administration ${ }^{1-6}$; the facts related to a mitochondrial dysfunction during local anesthetic cardiotoxicity 13-24,44-51, and the key role of cardiolipin in mitochondrial functioning ${ }^{35-40}$, we therefore propose that the interaction between the local anesthetic and cardiolipin determines the toxic effect of local anesthetics on cardiomyocytes by interfering with the mitochondrial energy metabolism. This interaction model can be produced with methods of theoretic and structural biology which gives insights of the potential interacting relation and clues for designing interventional drugs for such inadvertent events.

\section{ACKNOWLEDGEMENT}

The authors are indebted to Mrs. YuFeng Wang, MA, Senior Librarian and Staff Literature Archiving, at the Library of Southeast University Medical School, Nanjing, China, for her help in performing literature search. 


\section{REFERÊNCIAS / REFERENCES}

01. Howell BA, Chauhan A - Bupivacaine binding to pegylated liposomes. Anesth Analg, 2009;109:678-682.

02. Clark MK - Lipid emulsion as rescue for local anesthetic-related cardiotoxicity. J Perianesth Nurs 2008; 23:111-117.

03. Mazoit JX, Le Guen R, Beloeil H et al. - Binding of long-lasting local anesthetics to lipid emulsions. Anesthesiology, 2009;110:380386.

04. McCutchen T, Gerancher JC - Early intralipid therapy may have prevented bupivacaine-associated cardiac arrest. Reg Anesth Pain Med, 2008;33:178-180.

05. Sirianni AJ, Osterhoudt KC, Calello DP et al. - Use of lipid emulsion in the resuscitation of a patient with prolonged cardiovascular collapse after overdose of bupropion and lamotrigine. Ann Emerg Med 2008; 51:412-415.

06. Felice K, Schumann $\mathrm{H}$ - Intravenous lipid emulsion for local anesthetic toxicity: a review of the literature. J Med Toxicol 2008; 4:184191.

07. Leskiw $U$, Weinberg GL - Lipid resuscitation for local anesthetic toxicity: is it really lifesaving? Curr Opin Anaesthesiol, 2009; 22:667-671

08. Marwick PC, Levin Al, Coetzee AR - Recurrence of cardiotoxicity after lipid rescue from bupivacaine-induced cardiac arrest. Anesth Analg, 2009;108:1344-1346.

09. Weinberg GL - Limits to lipid in the literature and lab: what we know, what we don't know. Anesth Analg, 2009;108:1062-1064.

10. Hicks SD, Salcido DD, Logue ES et al. - Lipid emulsion combined with epinephrine and vasopressin does not improve survival in a swine model of bupivacaine-induced cardiac arrest. Anesthesiology, 2009;111:138-146.

11. de Jong RH - Lipid infusion for cardiotoxicity: promise? Yes-panacea? Not. Anesthesiology, 2007;106:635-636.

12. Mather LE, Chang DH - Cardiotoxicity with modern local anaesthetics: is there a safer choice? Drugs, 2001;61:333-342.

13. Vanderkooi G, Chazotte B, Biethman R - Temperature dependence of anesthetic effects on succinate oxidase activity in uncoupled submitochondrial particles. FEBS Lett, 1978;90:21-23.

14. Chazotte B, Vanderkooi G - Multiple sites of inhibition of mitochondrial electron transport by local anesthetics. Biochim Biophys Acta 1981;636:153-161.

15. Saeki H, Nozawa $\mathrm{Y}$, Shimonaka $\mathrm{H}$ et al. - Effects of anesthetics, dibucaine and methoxyflurane on the ATPase activity and physical state of Tetrahymena surface membranes. Biochem Pharmacol, 1979;28:1095-1098.

16. Vanderkooi G, Shaw J, Storms C et al. - On the mechanism of action of anesthetics. Direct inhibition of mitochondrial F1-ATPase by $n$-butanol and tetracaine. Biochim Biophys Acta, 1981; 636:200203. 
17. Adade AB, O'Brien KL, Vanderkooi G - Temperature dependence and mechanism of local anesthetic effects on mitochondrial adenosinetriphosphatase. Biochemistry, 1987;26:7297-7303.

18. Azzi A, Scarpa A - Inhibition of K+ transport in liver mitochondria. Biochim Biophys Acta 1967; 135:1087-1088.

19. Selwyn MJ, Fulton DV, Dawson AP - Inhibition of mitochondrial anion permeability by local anaesthetics. FEBS Lett, 1978;96:148-151.

20. Barritt GJ - Inhibition by local anaesthetics of anion transport in isolated rat heart mitochondria. Biochem Pharmacol, 1979;28: 1017-1021.

21. Dawson AP, Selwyn MJ, Fulton DV - Inhibition of Ca2+ efflux from mitochondria by nupercaine and tetracaine. Nature, 1979; 277:484-486.

22. Garlid KD, Nakashima RA - Studies on the mechanism of uncoupling by amine local anesthetics. Evidence for mitochondrial proton transport mediated by lipophilic ion pairs. J Biol Chem 1983;258:7974-7980.

23. Dabadie $P$, Bendriss $P$, Erny $P$ et al. - Uncoupling effects of local anesthetics on rat liver mitochondria. FEBS Lett, 1987;226:77-82.

24. Terada $\mathrm{H}$, Shima O, Yoshida $\mathrm{K}$ et al. - Effects of the local anesthetic bupivacaine on oxidative phosphorylation in mitochondria. Change from decoupling to uncoupling by formation of a leakage type ion pathway specific for $\mathrm{H}+$ in cooperation with hydrophobic anions. J Biol Chem, 1990; 265:7837-7842.

25. Houtkooper RH, Vaz FM - Cardiolipin, the heart of mitochondrial metabolism. Cell Mol Life Sci 2008;65:2493-2506.

26. Chicco AJ, Sparagna GC - Role of cardiolipin alterations in mitochondrial dysfunction and disease. Am J Physiol Cell Physiol, 2007;292:C33-44.

27. Önyüksel $H$, Sethi $V$, Weinberg $G L$ et al. - Bupivacaine, but not lidocaine, disrupts cardiolipin-containing small biomimetic unilamellar liposomes. Chem Biol Interact, 2007;169:154-159.

28. Mulroy MF - Systemic toxicity and cardiotoxicity from local anesthetics: incidence and preventive measures. Reg Anesth Pain Med, 2002;27:556-561.

29. Farid IS, Hernandez-Popp V, Youssef GN et al. - Bupivacaine induces transient neurological symptoms after subarachnoid block. Pain Pract 2002;2:53-55.

30. de La Coussaye JE, Aya AG, Eledjam JJ - Neurally-mediated cardiotoxicity of local anesthetics: direct effect of seizures or of local anesthetics? Anesthesiology, 2003;98:1295-1296.

31. Gould DB, Aldrete JA - Bupivacaine cardiotoxicity in a patient with renal failure. Acta Anaesthesiol Scand, 1983;27:18-21.

32. Conklin KA, Ziadlou-Rad F - Bupivacaine cardiotoxicity in a pregnant patient with mitral valve prolapse. Anesthesiology, 1983;58:596.

33. Soltesz EG, van Pelt F, Byrne JG. - Emergent cardiopulmonary bypass for bupivacaine cardiotoxicity. J Cardiothorac Vasc Anesth, 2003;17:357-358.

34. Ho AM, Dion PW, Karmakar MK et al. - Estimating with confidence the risk of rare adverse events, including those with observed rates of zero. Reg Anesth Pain Med, 2002;27:207-210.

35. Lambeth JD - Cytochrome P-450scc. Cardiolipin as an effector of activity of a mitochondrial cytochrome P-450. J Biol Chem 1981; 256:4757-4762.

36. Claypool SM, Oktay $Y$, Boontheung $P$ et al. - Cardiolipin defines the interactome of the major ADP/ATP carrier protein of the mitochondrial inner membrane. J Cell Biol, 2008;182:937-950.

37. Chen S, Tarsio M, Kane PM et al. - Cardiolipin mediates cross-talk between mitochondria and the vacuole. Mol Biol Cell, 2008;19:50475058.

38. Müller M, Moser R, Cheneval D et al. - Cardiolipin is the membrane receptor for mitochondrial creatine phosphokinase. J Biol Chem 1985;260:3839-3843.

39. Petrosillo G, Portincasa P, Grattagliano I et al. - Mitochondrial dysfunction in rat with nonalcoholic fatty liver: involvement of complex I, reactive oxygen species and cardiolipin. Biochim Biophys Acta 2007; 1767:1260-1267.

40. Sparagna GC, Chicco AJ, Murphy RC et al. - Loss of cardiac tetralinoleoyl cardiolipin in human and experimental heart failure. J Lipid Res, 2007;48:1559-1570.
41. Sztark F, Malgat M, Dabadie $P$ et al. - Comparison of the effects of bupivacaine and ropivacaine on heart cell mitochondrial bioenergetics. Anesthesiology, 1998;88:1340-1349.

42. Sztark F, Nouette-Gaulain K, Malgat $M$ et al. - Absence of stereospecific effects of bupivacaine isomers on heart mitochondrial bioenergetics. Anesthesiology, 2000;93:456-462.

43. Nouette-Gaulain K, Forestier F, Malgat M et al. - Effects of bupivacaine on mitochondrial energy metabolism in heart of rats following exposure to chronic hypoxia. Anesthesiology, 2002;97:1507-1511.

44. Grouselle M, Tueux O, Dabadie P et al. - Effect of local anaesthetics on mitochondrial membrane potential in living cells. Biochem J, 1990;271:269-272.

45. Porter JD, Edney DP, McMahon EJ et al. - Extraocular myotoxicity of the retrobulbar anesthetic bupivacaine hydrochloride. Investig Ophthalmol Vis Sci, 1988;29:163-174.

46. Steer JH, Mastaglia FL, Papadimitriou JM et al. - Bupivacaineinduced muscle injury. The role of extracellular calcium. J Neurol Sci, 1986;73:205-217.

47. Sztark F, Tueux O, Erny P et al. - Effects of bupivacaine on cellular oxygen consumption and adenine nucleotide metabolism. Anesth Analg, 1994;78:335-339.

48. Bernardi $P$ - Mitochondrial transport of cations: channels, exchangers, and permeability transition. Physiol Rev, 1999;79:11271155.

49. Irwin W, Fontaine E, Agnolucci L et al. - Bupivacaine myotoxicity is mediated by mitochondria. J Biol Chem 2002; 277:12221-12227.

50. David JS, Ferreti C, Amour J et al. - Effects of bupivacaine, levobupivacaine and ropivacaine on myocardial relaxation. Can J Anaesth, 2007;54:208-217.

51. Nouette-Gaulain K, Sirvent P, Canal-Raffin M et al. - Effects of intermittent femoral nerve injections of bupivacaine, levobupivacaine, and ropivacaine on mitochondrial energy metabolism and intracellular calcium homeostasis in rat psoas muscle. Anesthesiology, 2007;106:1026-1034.

52. Kowaltowski AJ, Naia-da-Silva ES, Castilho RF et al. - Ca2+- stimulated mitochondrial reactive oxygen species generation and permeability transition are inhibited by dibucaine or Mg2+. Arch Biochem Biophys, 1998;359:77-81.

53. Arita K, Utsumi T, Kato $A$ et al. - Mechanism of dibucaine-induced apoptosis in promyelocytic leukemia cells (HL-60). Biochem Pharmacol, 2000;60:905-915.

54. Werdehausen R, Braun S, Essmann F et al. - Lidocaine induces apoptosis via the mitochondrial pathway independently of death receptor signaling. Anesthesiology, 2007;107:136-143.

55. Corman SL, Skledar SJ - Use of lipid emulsion to reverse local anesthetic-induced toxicity. Ann Pharmacother, 2007;41:18731877.

56. Edelman LB, Ripper R, Kelly $\mathrm{K}$ et al. - Metabolic context affects hemodynamic response to bupivacaine in the isolated rat heart. Chem Biol Interact, 2008;172:48-53.

57. Malhotra A, Xu Y, Ren M et al. - Formation of molecular species of mitochondrial cardiolipin 1. A novel transacylation mechanism to shuttle fatty acids between sn-1 and sn-2 positions of multiple phospholipid species. Biochim Biophys Acta 2009;1791:314320.

58. Schlame M - Formation of molecular species of mitochondrial cardiolipin 2. A mathematical model of pattern formation by phospholipid transacylation. Biochim Biophys Acta 2009;1791:321-325.

59. Beranek A, Rechberger $\mathrm{G}, \mathrm{Knauer} \mathrm{H}$ et al. - Identification of a cardiolipin-specific phospholipase encoded by the gene CLD1 (YGR110W) in yeast. J Biol Chem, 2009;284:11572-11578.

60. Sorice M, Manganelli V, Matarrese $P$ et al. - Cardiolipin-enriched raft-like microdomains are essential activating platforms for apoptotic signals on mitochondria. FEBS Lett, 2009;583:2447-2450.

61. Schug ZT, Gottlieb E - Cardiolipin acts as a mitochondrial signalling platform to launch apoptosis. Biochim Biophys Acta, 2009;1788:2022-2031.

62. Takashi T, Inoue K, Nojima S - Immune reactions of liposomes containing cardiolipin and their relation to membrane fluidity. J Biochem (Tokyo), 1980;87:679-685. 
63. Shimooka $\mathrm{T}$, Seto $\mathrm{S}$, Terada $\mathrm{H}$ - Increase in water permeability of negatively charged liposomal membrane by local anesthetics. Chem Pharm Bull (Tokyo) 1992;40:1880-1882.

64. Wilschut J, Holsappel M, Jansen R - Ca2+-induced fusion of cardiolipin/phosphatidylcholine vesicles monitored by mixing of aqueous contents. Biochim Biophys Acta, 1982;690:297-301.

65. Larsen SW, Frost AB, Østergaard J et al. - On the mechanism of drug release from oil suspensions in vitro using local anesthetics as model drug compounds. Eur J Pharm Sci, 2008;34:37-44.

66. Dahlberg M, Maliniak A - Molecular dynamics simulations of cardiolipin bilayers. J Phys Chem B, 2008;112:11655-11663.
67. Sinibaldi F, Fiorucci L, Patriarca A et al. - Insights into cytochrome ccardiolipin interaction. Role played by ionic strength. Biochemistry, 2008;47:6928-6935.

68. Sani MA, Dufourc EJ, Gröbner G - How does the Bax-alpha1 targeting sequence interact with mitochondrial membranes? The role of cardiolipin. Biochim Biophys Acta, 2009;1788:623-631.

69. Muhonen J, Holopainen JM, Wiedmer SK - Interactions between local anesthetics and lipid dispersions studied with liposome electrokinetic capillary chromatography. J Chromatogr A, 2009;1216:3392-3397.

70. Padera R, Bellas E, Tse JY et al. - Local myotoxicity from sustained release of bupivacaine from microparticles. Anesthesiology, 2008;108:921-928. 